\title{
Widowhood and mortality risk of older people in rural China: do gender and living arrangement make a difference?
}

\author{
Huijun Liu ${ }^{1 *}$, Quanbao Jiang ${ }^{1}$ and Marcus W. Feldman ${ }^{2}$ \\ ${ }^{1}$ Center for Ageing and Health Research, School of Public Policy and Administration, Xi'an Jiaotong \\ University, Xi'an, China and ${ }^{2}$ Morrison Institute for Population and Resource Studies, Stanford University, \\ Stanford, California, USA \\ *Corresponding author. Email: liuhuij@mail.xjtu.edu.cn
}

(Accepted 12 March 2019; first published online 10 April 2019)

\begin{abstract}
Increased mortality after spousal bereavement has been observed in many populations. Few studies have investigated the widowhood effect in a traditional culture where the economy is underdeveloped. The reasons for the widowhood effect and its gender dynamic are not well understood. In this study, we assessed whether the widowhood-associated excess mortality exists and differs by gender and living arrangement in rural China. We used a six-wave panel of data derived from rural people over 60 years old in the Chaohu region of China. Cox regression analyses suggest that there was a positive effect of spousal loss on mortality for older rural Chinese and this effect was gender different. Our findings also suggest that living with adult children after spousal loss played a protective role in reducing the risk of older men's death, though it tended to increase older men's mortality risk in general.
\end{abstract}

Keywords: widowhood; mortality; living arrangements; rural

\section{Introduction}

China's population is ageing at a faster rate than most Western societies due to falling fertility and increasing longevity. Together with population ageing and the age gap in life expectation, the widowed elders have attracted more attention due to the increasing number of widowed elders and the important role of the spouse in oldage support in China. It is estimated that by 2050, the population of widowed elders (aged 60 years and older) in China will reach 118 million, and the majority of the widowed elders reside in rural areas (Wang and Ge, 2013). Marriage has long been recognised as a fundamental social institution that provides important protective barriers against stressful consequences of external threats (Umberson, 1992). Its effects on health and mortality have been systematically examined by sociologists and demographers ( $\mathrm{Hu}$ and Goldman, 1990; Bulanda et al., 2016). As a common 
and inevitable major life event among married people, spousal loss has also been widely recognised as one of life's most potent stressors.

It has been suggested that people experiencing the loss of a spouse are at a greater risk of dying than those whose partner is still living (Schaefer et al., 1995; Shor et al., 2012; Prior et al., 2018). Spousal loss is believed to contribute to excess mortality than those in marriage, especially in the near term, by stress responses such as immune system disruption (Gerra et al., 2003) and cardiovascular effects (Buckley et al., 2010; Stahl et al., 2016), or by triggering health-hazardous behaviours such as self-neglect or substance abuse (Mellström et al., 1982). The rapid effects of grief have been consistently confirmed in most empirical studies, and the increased mortality risk was reported highest within the first six months following spousal loss (Martikainen and Valkonen, 1996a; Shah et al., 2012; Moon et al., 2014). However, evidence suggests that the increased mortality of the surviving spouse is not due to specific incidents or worsening chronic health conditions following bereavement (Shah et al., 2012; Einiö and Martikainen, 2019). Many researchers have argued that elevated mortality risk following spousal loss is not necessarily a result of the bereavement itself, and it was difficult to distinguish the widowhood effect from those of spousal similarity, selection and shared environmental exposure (Schaefer et al., 1995; Boyle et al., 2011). It is reasonable to assume that people with similar characteristics or lifestyles tend to marry each other and share many aspects of their daily life after marriage. If any of these similarities are detrimental to health, both spouses run a risk of early death. A number of studies have examined the possible contribution of spousal similarities to the excess mortality by adjusting confounders or distinguishing causes of death. Although the magnitude of the widowhood effect was attenuated after adjustment of lifestyle factors (Mellström et al., 1982; Skulason et al., 2012), some studies confirmed that loss of a spouse was independently associated with excess mortality (Schaefer et al., 1995; Williams et al., 2011). Some evidence indicated differential mortality risk by cause of death and excess mortality was found to be markedly higher when the spouse dies from accidental, violent or alcohol-related causes (Martikainen and Valkonen, 1996b; Shah et al., 2013). However, only a few more widowed individuals than expected died from the same cause-group as their spouses (Brenn and Ytterstad, 2016). These inconsistent findings suggest the link between widowhood and causes of death is not yet clear.

Although a few studies report no significant effect of the widowhood on death risk (Helsing and Szklo, 1981; Jones et al., 1984; Stahl et al., 2016), and the magnitude of the effect varies substantially, considerable excess mortality risk among bereaved older people than their married counterpart was observed in most studies. It is now generally accepted that spousal loss is causal although the effect is relatively small and short-lived (Martikainen and Valkonen, 1996a). The variability in findings was partly attributable to differences in sample sizes, geographic regions and levels of statistical adjustment (Shor et al., 2012). It has been argued that this differential responsiveness to stress can be explained in terms of both individual and environmental differences (Kessler and Cleary, 1980). Given the public health importance of health inequalities according to socio-economic status, spousal loss may lead to differential exposure to risk for different sub-populations or in different countries, and marriage may also play a different role in the context of different 
cultural values and social systems. Whether the effect of bereavement on mortality varies between socio-economic groups was addressed in recent studies, and the evidence suggests that the bereaved with higher status had more, rather than less, excess mortality, although higher-status individuals are associated with lower mortality in general (Manor and Eisenbach, 2003; Boyle et al., 2011; Shah et al., 2012). The impacts of spousal bereavement on mortality risk also varied between urban and rural areas (Wright et al., 2015). One study also examined whether the widowhood effect was culture-specific by distinguishing the race of partners, and showed that whites in endogamous marriages suffered a large widowhood effect, but this effect was not evident among blacks (Elwert and Christakis, 2006). However, most widowhood studies were conducted in Western societies, and primarily with European samples (Li et al., 2005); relatively few explored the widowhood effect in developing countries, such as China, with substantially different cultural values and social systems from Western societies.

Based on the division of gender roles, marriage is expected to be more beneficial for men than for women (Umberson, 1992), which suggests that it is harder for men to adjust to widowhood because they are more likely than women to rely on their spouse to maintain social interactions and carry out domestic tasks (Lee et al., 2001). The gender dynamic for mortality excess following bereavement has been tested in a number of studies. Those studies suggest that there is a causal effect only among men (Martikainen and Valkonen, 1996a; Stahl et al., 2016). However, other studies have argued that the relationship is causal for both sexes (Boyle et al., 2011; Ytterstad and Brenn 2015), and the bereavement effects on mortality is similar for males and females (Manor and Eisenbach 2003; Santacroce et al., 2018). Family function and expected roles of husbands and wives vary across countries and regions, with different rates and meanings of widowhood, suggesting that widowhood effects and gender differences are very likely to be culture-specific. However, little information is available on whether the widowhood-associated mortality risk varies by gender in developing countries. A study in Taiwan examined gender differences in widowhood effects among community-dwelling elders between 1989 and 2000, and suggested that spousal loss-related mortality was increased by lowering the probability of death at baseline ascertainment for widowers, while the risks for females seemed rather homogeneous (Fang et al., 2012). Another study concerning the gender dynamic of the widowhood effect in Chinese older-oldest between 1998 and 2001 suggested that the negative effect was higher among women than among men $(\mathrm{Gu}, 2003)$. These disparate results suggest the need for further studies that examine gender differences in widowhood effects in more birth cohorts as gender role norms change, especially those concerning the effect of gender-age interaction. Previous studies have shown the widowhood effect was more pronounced for younger cohorts than older ones (Martikainen and Valkonen, 1996b; Brenn and Ytterstad, 2016), and dropped with age especially among men (Ytterstad and Brenn, 2015).

In most countries, especially those with underdeveloped social and institutional support systems, adult children have long been the primary source of long-term care for their older parents and co-reside with them as necessary (Pezzin et al., 2007; Ward, 2008). Numerous studies have examined the association between structural family support and elders' health in a wide variety of countries. 
However, they have produced inconsistent findings. Some studies suggested that living alone had a higher death risk (Lund et al., 2002; Pimouguet et al., 2016). However, a protective effect of living alone on mortality risk among elders was seen in other studies (Walter-Ginzburg et al., 2002; Herm et al., 2016). Studies examining the association of living arrangement and death risk in China found that living with adult children increased mortality risk, especially among those elders with cardiovascular diseases (Li et al., 2009; Liu et al., 2015). Family support has also been identified as a major contributor that buffers the effects of widowhood on health (Li et al., 2005). Empirical evidence from the United States of America and Japan indicated that structural and functional family support moderated the increase in psychological distress associated with widowhood (Silverstein and Bengtson, 1994; Tiedt et al., 2016), and affectionate relations with adult children offset mortality risk associated with spousal death (Silverstein and Bengtson, 1991). Two studies in Italy and the Pacific found that living alone plays a protective role for death risk of elders following spousal loss (McNally, 2003; Pizzetti and Manfredini, 2008). Studies in Asian countries have found that living with children was psychologically beneficial for widowed women (Do and Malhotra, 2012) and support from children buffers the effect of widowhood on depression among both older men and women (Li et al., 2005). However, it is still unclear whether family support buffers the effects of widowhood on mortality risk in Asian countries and whether the dynamic differs by gender.

The main purpose of this paper, therefore, is to investigate the widowhood effect on death risk and whether living arrangement buffers the effect of spousal loss on mortality in the strong family-oriented Chinese culture, by distinguishing those who are widowed before baseline from those who become widowed in follow-up. In particular, we investigate gender difference in mortality following death of a spouse, and whether the effect of widowhood and its gender dynamic varies by cohort, cause of death and survey period.

\section{Data, measures and method}

\section{Data collection}

The data used in this longitudinal study come from the Well-being of Elderly Survey in Anhui Province (WESAP). As a joint project between Xi'an Jiaotong University and the University of Southern California, the survey has been conducted every three years between 2001 and 2015 in rural townships in Chaohu, Anhui province. Using a stratified multi-stage method, potential respondents were from 72 randomly selected villages within six rural townships in the Chaohu region, and residents aged 60 and older were identified in selected villages and randomly chosen from household rosters, with a proportionate over-sampling of people aged 75 and older. A questionnaire survey was administered to the subjects over 60 years old in the respondents' homes. Of the 1,800 elders identified as eligible respondents, 1,715 completed the baseline survey in 2001. All of the follow-up surveys include re-interviews with surviving participants, and a cohort of 420 individuals aged 60-68 replacing deceased and lost-to-follow-up elders was added to the sample in 2009. Among the six waves of data, 225 missing 
Table 1. Characteristics of the sample and gender difference

\begin{tabular}{|llll}
\hline Variables & Whole sample & Older men & Older women \\
\hline & & Percentages or mean values $(S D)$ \\
\hline Age & $71(0.16)$ & $70(0.21)$ & $72(0.23)^{\star \star \star}$ \\
\hline Education (literate) & 26.83 & 44.29 & $9.84^{\star \star \star}$ \\
\hline Household income (Yuan) & $1,551(61.78)$ & $1,896(97.31)$ & $1,216(75.81)^{\star \star \star}$ \\
\hline Occupation (farmer) & 93.80 & 92.10 & $95.45^{\star \star \star}$ \\
\hline Self-reported health & $2.26(0.02)$ & $2.56(0.02)$ & $1.96(0.02)^{\star \star \star}$ \\
\hline Onset of chronic disease & 76.27 & 71.76 & $80.65^{\star \star \star}$ \\
\hline Number of children & $3.81(0.03)$ & $3.80(0.05)$ & $3.81(0.04)$ \\
\hline Living arrangement: & & & $*$ \\
\hline Living alone (or only couple) & 51.05 & 52.36 & 49.76 \\
\hline Living with children & 28.89 & 25.62 & 32.08 \\
\hline Living with others & 20.06 & 22.02 & 18.16 \\
\hline Marital status: & & & $\star \star$ \\
\hline Married & 49.86 & 63.65 & 36.44 \\
\hline Widowed before & 39.92 & 26.53 & 52.95 \\
\hline Widowed after & 10.22 & 9.82 & 10.61 \\
\hline Death & 38.00 & 38.82 & 37.21 \\
\hline Death to disease & 78.29 & 83.07 & $73.64^{\star \star \star}$ \\
\hline Death to other reason & 21.71 & 16.93 & $26.36^{\star \star \star}$ \\
\hline N & 1,829 & 902 & 927 \\
\hline
\end{tabular}

Note: SD: standard deviation.

Significance levels: ${ }^{\star} p<0.05,{ }^{\star \star} p<0.01,{ }^{\star \star *} p<0.001$ ( $t$-test $/ F$-test).

cases (mainly migrants) at the follow-up surveys were excluded. We also restricted the sample to respondents who were married or widowed with adult children at baseline and in follow-up, and those people who were divorced or never married at baseline surveys were excluded. Finally, the data-set used in the study included 1,829 older people aged $60+$ who were interviewed in 2001 or 2009 and their follow-up data. In addition, we constructed up to two longitudinal transition intervals (2001-2009, 2009-2015) for the observations. During the period 2001-2009, 1,413 correspondents were used for data analysis and 1,058 respondents were used for data analysis during the period 2009-2015.

Table 1 shows the characteristics of the total sample. The average age is 71 years old, with 49 per cent male. More than 70 per cent are illiterate, nearly 95 per cent are farmers. On average, they have four children, and usually two are sons. Less than 30 per cent of respondents live with at least one of their children. More than half live alone or only with their spouse. Almost 40 per cent of respondents were widowed before 2001 or 2009 and over 10 per cent lost their spouse during 
the follow-up surveys. Almost 40 per cent died in follow-up, and of these over 78 per cent died from known illnesses. For gender differences among respondents, only the number of children and death percentage do not exhibit significant differences. The female sample is older on average, more widowed, includes more farmers, is less educated and has lower annual household income. Among the deceased respondents, more men died from some illness. However, women reported worse health conditions including greater incidence of chronic diseases. In addition, a larger share of respondents who were widowed before 2001 or 2009 and who live with a child were women.

\section{Dependent variables}

Mortality risk refers to the likelihood of death of a respondent before follow-up contact. Information on survival status and date of death were collected for the deceased and the survivors in follow-up interviews. Survival status was coded as survival versus death. Survival time was counted as months from the date of the baseline interviews in 2001 or 2009 to the date of the survey in 2009 or 2015 for the survivors, and to the date at death for the deceased. Respondent's initial age in 2001 or 2009 was also controlled for survival analyses.

\section{Independent variables}

The key independent variables of interest are marital status and living arrangement. At baseline surveys and each follow-up wave of interviews, the respondents were asked their marital status. Given that those who died before baseline were no longer eligible for investigation of effects of widowhood, analyses limited to the sample of those who remained alive may result in mis-estimation of the effects of widowhood. We therefore divide marital status into widowed before (marital status was 'widowed' before the baseline survey), widowed after (marital status became 'widowed' during follow-up interviews) and married (marital status remained 'married' during the whole survey period). Marital status and its changes for the deceased were also collected from their children or other family members during the follow-up waves. Living arrangements were classified into one of the following three categories: living alone (or only with spouse), living with at least one adult child (including living with both spouse and children) and living with others. In this study, pre-bereaved living arrangements and those after widowhood are distinguished by including both widowed and married respondents at baseline surveys.

\section{Confounders}

Health status at baseline is included as a potential confounder, and was evaluated through a series of questions about chronic conditions and self-reported health. Incidence of chronic disease is assessed for 12 diseases including diabetes, hypertension, heart disease and stroke. 'Incidence of chronic disease' is scored as 1 if the respondent reports having one or more of the 12 diseases, and otherwise as 0 . Respondents were also asked to evaluate their health status by a single question: What do you think about your current health status? A four-point scale was 
provided for the responses. We have reverse-coded self-reported health so that higher values indicate better health.

Individual socio-economic status and demographic indicators, which had been identified as important factors of health outcome of older people, were also considered confounders in this study. Individual education, family income and occupation were used to assess socio-economic status. Educational attainment was coded as a dichotomous variable: 'illiterate' and 'literate (primary school and above)'; household income was assessed by the total amount of earnings of the individual and his/her spouse in the previous year, including pensions, and was transformed using $\ln +1$. Occupation was coded as a dichotomous variable: $0=$ 'others' and $1=$ 'farmers'. Demographic characteristics included gender and age. Age was assessed as a continuous variable. Gender was measured as female versus male $($ male $=1)$. Given that family members, especially children, are the most important resources for old-age support of elderly parents under the culture of filial piety, the number of children was also controlled as a continuous variable.

\section{Data analysis}

By distinguishing those widowed before baseline from those in follow-up, the bias due to the selection was estimated. Gender differences were also identified by gender-specific analysis. To explore the moderating effect of living arrangement, two models were used for these regression analyses: the main effects of status of widowhood and living arrangement on mortality risk of rural elders were examined in Model 1; and the moderating effect of living arrangement on widowhood and mortality risk were tested in Model 2. All the data analyses were completed using Stata 11.

\section{Results}

\section{Widowhood, living arrangement and mortality risk}

Table 2 shows the results of Cox regression for mortality risk of rural elders. Model 1 examined the associations between marital status, living arrangement and mortality without controlling potential confounders. It shows that the mortality risk of widowed elders who lost their spouse before baseline was significantly higher than for those with surviving spouses, but excess mortality of elders widowed in follow-up was not statistically significant. After controlling for the confounders (shown as Model 2), the mortality risk of widowed elders instead declined significantly more than that of those with surviving spouses, while the associations between living arrangement and mortality remained statistically significant. When the moderating effect of living arrangement was further controlled in Model 3, the main effects of marital status and living arrangement remained statistically significant, and the moderating effect was also significant. Although spouse loss and living alone (or only with spouse) were associated with lower mortality risk in general, the mortality risk of widowed elders was significantly higher when they lived only with their spouse before widowhood or live alone after widowhood. In 
Table 2. Cox hazard regression model for widowhood, living arrangement and mortality risk

\begin{tabular}{|c|c|c|c|}
\hline \multirow[b]{2}{*}{ Variables } & \multicolumn{3}{|c|}{ All widowed } \\
\hline & Model 1 & Model 2 & Model 3 \\
\hline Gender & & $1.66^{\star \star \star}$ & $1.67^{\star \star \star}$ \\
\hline Age & & $1.10^{\star \star \star}$ & $1.10^{\star \star \star}$ \\
\hline Education (Ref. Illiterate) & & 0.89 & 0.88 \\
\hline Family income & & $0.94^{\star * \star}$ & $0.94^{* * *}$ \\
\hline Occupation (Ref. Others) & & 1.00 & 1.01 \\
\hline Self-reported health & & $0.74^{\star \star \star}$ & $0.74^{\star \star \star}$ \\
\hline Chronic disease & & $1.20^{\star}$ & $1.21^{\star}$ \\
\hline Number of children & & 0.99 & 0.98 \\
\hline \multicolumn{4}{|l|}{ Marital status (Ref. Married): } \\
\hline Widowed before & $1.79^{\star \star \star}$ & $0.77^{\star \star}$ & $0.62^{\star \star \star}$ \\
\hline Widowed after & 1.06 & $0.72^{\star \star}$ & $0.44^{\star \star}$ \\
\hline \multicolumn{4}{|c|}{ Living arrangement (Ref. Living with children): } \\
\hline Living alone (or only couple) & $0.82^{\star \star}$ & $0.85^{\star}$ & $0.67^{\star \star}$ \\
\hline Living with others & $0.52^{\star \star \star}$ & $0.75^{\star \star}$ & $0.59^{\star \star}$ \\
\hline \multicolumn{4}{|l|}{ Interactions: } \\
\hline Widowed before $\times$ Living alone & & & $1.37^{\star}$ \\
\hline Widowed before $\times$ Living with others & & & 1.27 \\
\hline Widowed after $\times$ Living alone & & & $1.78^{\star}$ \\
\hline Widowed after $\times$ Living with others & & & $2.69^{\star \star}$ \\
\hline
\end{tabular}

Note: Ref.: reference category.

Significance levels: $\dagger p<0.1,{ }^{\star} p<0.05,{ }^{\star \star} p<0.01,{ }^{\star \star \star} p<0.001$.

addition, widowed elders who lost their spouse in follow-up also had a higher mortality risk when they lived with others before widowhood.

Among the controlled variables, only gender, age, family income and health condition were significantly correlated with mortality risk. Mortality risk was higher among men than among women. The death risk of older people increased with age and decreased as family income increased. Older people with good self-reported health were less likely to die than those with bad self-reported health. The onset of chronic disease was also significantly associated with higher mortality risk.

\section{Gender difference in widowhood, living arrangement and mortality risk}

Gender-specific analysis confirmed that there was a significant gender difference for the effects of widowhood and living arrangement on mortality (shown as Table 3). Model 1 examined the main effects of widowhood and living arrangement controlled for the potential cofounders. Less death risk after widowhood was more pronounced 
Table 3. Gender difference in Cox hazard regression model for widowhood, living arrangement and mortality risk

\begin{tabular}{|c|c|c|c|c|}
\hline \multirow[b]{2}{*}{ Variables } & \multicolumn{2}{|c|}{ Older men } & \multicolumn{2}{|c|}{ Older women } \\
\hline & Model 1 & Model 2 & Model 1 & Model 2 \\
\hline Age & $1.09^{\star \star \star}$ & $1.09^{\star \star \star}$ & $1.11^{\star \star \star}$ & $1.11^{\star \star \star}$ \\
\hline Education (Ref. Illiterate) & 0.91 & 0.90 & 0.82 & 0.82 \\
\hline Family income & $0.94^{\star \star \star}$ & $0.94^{\star \star \star}$ & $0.94^{\star \star \star}$ & $0.94^{\star \star \star}$ \\
\hline Occupation (Ref. Others) & 0.92 & 0.93 & 1.13 & 1.12 \\
\hline Self-reported health & $0.76^{\star \star \star}$ & $0.76^{\star \star \star}$ & $0.72^{\star \star \star}$ & $0.73^{\star \star \star}$ \\
\hline Chronic disease & $1.24 \dagger$ & $1.24 \dagger$ & 1.20 & 1.18 \\
\hline Number of children & 0.99 & 0.99 & 0.98 & 0.98 \\
\hline \multicolumn{5}{|l|}{ Marital status (Ref. Married): } \\
\hline Widowed before & 0.86 & $0.61^{\star \star}$ & $0.63^{\star *}$ & $0.66 \dagger$ \\
\hline Widowed after & 0.79 & $0.40^{\star \star}$ & $0.60^{\star *}$ & 0.57 \\
\hline \multicolumn{5}{|l|}{$\begin{array}{l}\text { Living arrangement (Ref. Living with } \\
\text { children): }\end{array}$} \\
\hline Living alone (or only couple) & $0.79^{*}$ & $0.57^{\star \star \star}$ & 0.92 & 1.01 \\
\hline Living with others & $0.71^{*}$ & $0.56^{\star *}$ & 0.82 & 0.68 \\
\hline \multicolumn{5}{|l|}{ Interactions: } \\
\hline Widowed before $\times$ Living alone & & $1.74^{\star}$ & & 0.86 \\
\hline Widowed before $\times$ Living with others & & 0.83 & & 1.40 \\
\hline Widowed after $\times$ Living alone & & $2.22^{*}$ & & 1.08 \\
\hline Widowed after $\times$ Living with others & & $4.91^{\star \star}$ & & 1.19 \\
\hline
\end{tabular}

Note: Ref.: reference category.

Significance levels: $\dagger p<0.1,{ }^{\star} p<0.05,{ }^{\star \star} p<0.01,{ }^{\star \star \star} p<0.001$.

among older women rather than among older men in Model 1. Compared with living alone or with others, living with at least one adult child significantly increased the death risk of older men but not of older women. When the moderating effect of living arrangement was considered in Model 2, the main effects of widowhood on mortality became statistically significant among older men while the main effect of becoming widowed in follow-up was no longer significant among older women. Both the main and moderating effects of living arrangement were significant among older men, which suggests that living alone after widowhood increases the chance of death for widowed men, although living alone was associated with a lower chance of death than living with adult children in general. In addition, living with others or only with a spouse before widowhood also increased the death risk of widowed men who lost their spouse within follow-up. However, both the main effect and moderating effect of living arrangement were not significant for older women. There is no significant gender difference in the effects of confounders. 


\section{Robustness check by age group, cause of death and survey period}

To examine whether our estimates are robust to age group, death causes and survey period, we classified the sample into adult elders ( $<75$ years old) and older elders $(75+$ years old), then distinguished those who died from disease and those who died from other causes, and finally divided our sample into two sub-groups, namely those interviewed between 2001 and 2009 and those interviewed between 2009 and 2015.

As Table 4 shows, the estimates for different cohorts based on age at baseline suggest that the effects of widowhood and living arrangement and their gender dynamics varied across cohorts. For adult elders, the mortality risk was not significantly different between married men and widowed men, and women bereaved before baseline had lower mortality risk than married women but the effect was no longer significant after controlling the moderating effect of living arrangement. Both the main effect and moderating effect of living arrangement were almost insignificant among adult elders and there was no gender difference in these estimates. For older elders, bereavement was significantly associated with women's lower mortality risk while only spousal loss in follow-up was significantly correlated with lower mortality risk of men. However, the estimate of widowed before baseline became significant among men and bereavement was no long significant among women when the moderating effect of living arrangement was controlled. Both the main effect and moderating effect of living arrangement were significant for men but not for women.

The estimates for different sub-samples divided by cause of death are also shown in Table 4, where we see that the impacts of widowhood and living arrangement depended on the causes of the respondent's death. Widowhood only significantly reduced the chance of death from disease and this was not significant for death risk from other reasons. The estimates of living arrangement were also inconsistent across sub-groups in terms of their statistical significance and effect magnitude among older men. However, the moderating effect of living alone remained consistent across these two sub-groups, which suggested that living alone could significantly increase all-cause death risk of widowed men.

As to the robustness check for different sub-groups classified according to the survey period, Table 4 shows that although the estimates of widowhood and living arrangement varied across the two survey periods in terms of their statistical significance and effect magnitude, the effect of widowhood and living alone or with others remained stable. The main effect of widowed in follow-up was greater during the period 2001-2009 than during 2009-2015. However, the risk estimates of death associated with living with adult children were greater during the period 2009-2015 than during 2001-2009. Though the moderating effect of living arrangement remained consistent across these two survey periods, the risk estimate of death associated with living alone among older men who lost their spouses in follow-up was only significant during the period 2001-2009.

\section{Conclusion and discussion}

Using data from a six-wave panel derived from rural elders over 60 years old, this study provides the first systematic evaluation of mortality after spousal loss and its gender difference in rural China. Our study extends bereavement studies conducted in Western developed countries to areas with underdeveloped economies and 
Table 4. Cox hazard regression model for robustness check by age group, cause of death and survey period

\begin{tabular}{|c|c|c|c|c|c|c|c|c|}
\hline & \multicolumn{2}{|c|}{ Older men } & \multicolumn{2}{|c|}{ Older women } & \multicolumn{2}{|c|}{ Older men } & \multicolumn{2}{|c|}{ Older women } \\
\hline \multicolumn{9}{|l|}{ Marital status (Ref. Married): } \\
\hline Widowed before & 0.76 & 0.69 & $0.57^{\star}$ & 0.66 & 0.85 & $0.43^{\star \star \star}$ & $0.66^{*}$ & 0.69 \\
\hline Living alone (or only couple) & 0.85 & 0.81 & 1.06 & 1.05 & $0.74^{*}$ & $0.34^{\star \star \star}$ & 0.88 & 0.95 \\
\hline Living with others & 0.76 & 0.72 & $0.55 \dagger$ & 0.54 & $0.68 \dagger$ & $0.41^{\star \star}$ & 1.08 & 0.94 \\
\hline \multicolumn{9}{|l|}{ Interactions: } \\
\hline Widowed before $\times$ Living alone & & 1.32 & & 0.74 & & $2.85^{\star \star \star}$ & & 0.92 \\
\hline Cause of death & \multicolumn{4}{|c|}{ Death from disease } & \multicolumn{4}{|c|}{ Death from other reasons } \\
\hline \multicolumn{9}{|l|}{ Marital status: } \\
\hline Widowed before & 0.84 & $0.62^{*}$ & $0.56^{\star \star \star}$ & $0.61 \dagger$ & 1.36 & 0.54 & 1.00 & 0.95 \\
\hline Widowed after & 0.77 & $0.44^{\star}$ & $0.57^{\star \star}$ & 0.55 & 1.38 & 0.18 & 0.82 & 0.74 \\
\hline \multicolumn{9}{|l|}{ Living arrangement: } \\
\hline Living alone (or only couple) & $0.75^{*}$ & $0.57^{\star \star \star}$ & 0.84 & 0.95 & 0.90 & $0.34^{*}$ & 0.99 & 1.02 \\
\hline
\end{tabular}


Table 4. (Continued.)

\begin{tabular}{|c|c|c|c|c|c|c|c|c|}
\hline & \multicolumn{2}{|c|}{ Older men } & \multicolumn{2}{|c|}{ Older women } & \multicolumn{2}{|c|}{ Older men } & \multicolumn{2}{|c|}{ Older women } \\
\hline & Model 1 & Model 2 & Model 1 & Model 2 & Model 1 & Model 2 & Model 1 & Model 2 \\
\hline Living with others & $0.65^{\star \star}$ & $0.55^{\star \star}$ & 0.76 & 0.67 & 0.93 & 0.43 & 0.96 & 0.44 \\
\hline \multicolumn{9}{|l|}{ Interactions: } \\
\hline Widowed before $\times$ Living alone & & $1.74^{\star}$ & & 0.82 & & $3.29^{\star}$ & & 0.94 \\
\hline Widowed before $\times$ Living with others & & 0.51 & & 1.33 & & 2.25 & & 2.65 \\
\hline Widowed after $\times$ Living alone & & 1.82 & & 1.03 & & $11.12^{\star}$ & & 1.11 \\
\hline Widowed after $\times$ Living with others & & $5.17^{\star \star}$ & & 1.10 & & $11.79 \dagger$ & & 1.57 \\
\hline Survey period & \multicolumn{4}{|c|}{$2001-2009$} & \multicolumn{4}{|c|}{$2009-2015$} \\
\hline \multicolumn{9}{|l|}{ Marital status: } \\
\hline Widowed before & 0.86 & $0.61^{\star}$ & $0.64^{\star}$ & 0.63 & 0.80 & $0.43^{*}$ & $0.58^{*}$ & 0.72 \\
\hline Widowed after & $0.72 \dagger$ & $0.35^{\star \star}$ & $0.53^{\star \star}$ & 0.53 & 0.82 & $0.33 \dagger$ & 0.77 & 0.63 \\
\hline \multicolumn{9}{|l|}{ Living arrangement: } \\
\hline Living alone (or only couple) & 0.95 & $0.67^{\star}$ & 0.99 & 1.02 & $0.60^{\star}$ & $0.38^{\star \star}$ & 0.80 & 0.97 \\
\hline Living with others & 0.86 & $0.69 \dagger$ & 1.01 & 0.73 & $0.53^{*}$ & $0.35^{\star \star}$ & $0.55 \dagger$ & 0.62 \\
\hline \multicolumn{9}{|l|}{ Interactions: } \\
\hline Widowed before $\times$ Living alone & & $1.80^{\star}$ & & 0.94 & & $2.28^{\star}$ & & 0.75 \\
\hline Widowed before $\times$ Living with others & & 0.75 & & 1.64 & & 1.42 & & 0.79 \\
\hline Widowed after $\times$ Living alone & & $2.52^{\star}$ & & 1.00 & & $2.64 \dagger$ & & 1.22 \\
\hline Widowed after $\times$ Living with others & & $4.62^{\star \star}$ & & 1.04 & & $8.84^{\star}$ & & 1.56 \\
\hline
\end{tabular}


traditional culture of filial piety, and provides greater understanding of the role of family support on mortality risk of rural elders.

\section{The effect of bereavement in China}

Our results confirm the socio-economic differences in the effect of bereavement on mortality found in previous studies (Elwert and Christakis, 2006; Shah et al., 2012; Wright et al., 2015). However, unlike other studies on the effect of bereavement on mortality in Western societies and in urban areas of China (Fang et al., 2012), our findings suggest that spousal loss was associated with lower rather than higher mortality risk of rural elders in China, after adjusting for social-demographic characteristics and health condition at baseline. A possible explanation is that the rural elderly, especially in their heavily dependent old age, have less to lose from bereavement while multiple supportive ties and meaningful family roles in China may protect the rural elderly from the deleterious effects of spousal loss (Li et al., 2005). Different from the strong public institutions for old-age support and autonomous values in Western societies, family-based assistance acting as functional solidarity has long been the primary source of support for the rural elderly in China. When rural elders become widowed, they are likely to receive more care and help from their adult children (Huang et al., 2018), which can compensate for the emotional vacuum and financial or instrumental deficit after spousal loss. Support from adult children has been confirmed to offset the decline in positive mood and mortality risk associated with spousal loss (Silverstein and Bengtson, 1991, 1994). The relief model of care-giving burden (Schulz et al., 1997; Keene and Prokos, 2008) may also shed some light on the positive effect of widowhood found in our study. A spouse is not only a resource but also a demander of care support. Care-giving has been proved to be a stressful situation for care-givers in previous studies (Capistrant et al., 2012; Nielsen et al., 2016), especially when the activities exceed the care-giver's abilities and resources, which may lead to health decline or higher mortality risk for the married elders with spouses in ill health. This situation may resolve after the death of a spouse, resulting in better health for the bereaved care-givers. Previous evidence has confirmed a lower rate of depression of care-givers after spousal death (Keene and Prokos, 2008) and worse physical and mental health for individuals approaching widowhood but no difference after widowhood (Vable et al., 2015; Santacroce et al., 2018).

\section{Gender differences in the effect of bereavement}

It has been suggested in most previous studies that the mortality risk associated with spousal loss is gender-dependent (Martikainen and Valkonen, 1996a; Stahl et al., 2016) and the widowhood effect is more prominent in male elders (Ytterstad and Brenn, 2015). In this study, we found that spousal loss was correlated with reduced mortality of elder women compared to elder men after adjustment of confounders. However, the positive effect of widowhood on death risk was more profound in elder men after controlling for the moderating effect of living arrangement. These findings may suggest that the widowhood effect is adjusted by a gender-dependent pattern of living arrangement after widowhood; that is, more widows than widowers are likely to live with adult children. Role theory, which has been used to explain the 
pathways between bereavement and mortality (Gove, 1984), may also explain the gender dynamics in the effect of bereavement. It has been suggested that men's main source of vulnerability after spousal loss stems from loneliness and difficulty in household management, while women are more likely to experience financial hardship (Umberson et al., 1992). Given that financial support is more constrained than other kinds of help in underdeveloped rural areas, financial dependence may place widows rather than widowers who have just been relieved of care-giving burden at greater risk. However, to avoid financial strain, widows are more likely to live with their adult children after spousal loss. Our robustness check by age group, cause of death and survey period confirmed the gender difference in the relative risk of mortality after spousal loss, and further showed that the gender dynamic of the widowhood effect seems to depend not only on age but also on cause of death. Spousal loss is associated with less mortality risk among male older elders and tends to reduce men's risk of death from disease, which may suggest that older elders, especially those who have a disease, may suffer more care-giving burden between couples. Gender difference in the widowhood effect is almost consistent across different survey periods, which suggests that caring for an ill spouse is still a big burden in current rural China, although some pilot projects on institutional old-age support (e.g. New Rural Social Pension Insurance; Senior Center at Village) have been initiated since 2009 and generally benefit rural elders' quality of life (Liu et al., 2015).

\section{The role of living arrangement}

In agreement with previous studies evaluating the effect of living arrangement on mortality risk in China (Li et al., 2009; Liu et al., 2015), our study confirmed that living with adult children increased the mortality risk of older men in rural China. However, different from previous findings that living alone played a protective role for elders' risk of death following spouse loss (McNally, 2003; Pizzetti and Manfredini, 2008), our findings suggest that living alone increased spousal loss-related mortality among older men. These findings suggest that living with adult children after spousal loss plays an important role in reducing death risk of older men in rural China, though it tended to increase older men's mortality risk in general. Additionally, this study also found that living only with a spouse or with others before widowhood also increased spousal loss-related mortality among older men, which may also confirm the relief model of care-giving burden. Robustness check by age group, cause of death and survey period further showed that the role of living arrangement and its gender difference was largely consistent across different causes of death and survey periods, but apparently varied across different age groups. Living alone significantly increases widowers' death risk irrespective of the cause of death and survey period. In addition, living with adult children is more likely to generally increase the death risk of men aged over 75 years but protect them from increased death risk after spousal loss. This may be attributed to the increasing dependence of older men on their family support as they age.

\section{Limitations}

A prospective and two-stage follow-up design with a representative community sample of rural elderly in China is well suited to investigating the widowhood effect. 
Distinguishing widowhood at baseline from those widowed in follow-up may also reduce potential bias resulting from selection and competing risks between death and widowhood. Some limitations warrant caution when interpreting our findings. Given the great regional heterogeneity in economic development, a major limitation of this study is that our data come from a well-defined area of central China, which is thought to typify the social and cultural conditions of poor rural areas. The extent to which our findings can be generalised to other rural elderly in China needs to be addressed by replicating the study in other rural areas. Another data limitation pertains to the lack of information concerning deceased spouses, including marriage quality and cause of death. Both are likely to be confounders of the observed relationship between individual widowhood and mortality. Furthermore, while our models control for socio-economic status and health conditions, data limitations prohibited us from considering health behaviour, which is likely to influence mortality and confound the relationship between widowhood and mortality. However, this may not be a serious problem with our analysis because health behaviour is an indicator of health condition, and the latter was controlled in our models as a confounder. Finally, changes in living arrangements between baseline and follow-up waves were not included, although a few respondents changed their living arrangements during the follow-up.

Acknowledgements. Data collection for this study was possible thanks to the joint financial support of the National Nature Science Foundation of China, Ministry of Education Foundation of China and Nature Science Foundation of Shaanxi Province. We are very grateful for the funding.

Financial support. This work was supported by the National Nature Science Foundation of China (grant number 71573207); the Ministry of Education Foundation of China (grant number 18JHQ073); and the Nature Science Foundation of Shaanxi Province (grant number 2016JM7006).

Conflict of interest. The authors declare no potential conflicts of interest with respect to the research, authorship and/or publication of this article.

\section{References}

Boyle PJ, Feng Z and Raab GM (2011) Does widowhood increase mortality risk? Testing for selection effects by comparing causes of spousal death. Epidemiology 22, 1-5.

Brenn T and Ytterstad E (2016) Increased risk of death immediately after losing a spouse: cause-specific mortality following widowhood in Norway. Preventive Medicine 89, 251-256.

Buckley T, McKinley S, Tofler G and Bartrop R (2010) Cardiovascular risk in early bereavement: a literature review and proposed mechanisms. International Journal of Nursing Studies 47, 229-238.

Bulanda JR, Brown JS and Yamashita T (2016) Marital quality, marital dissolution, and mortality risk during the later life course. Social Science \& Medicine 165, 119-127.

Capistrant BD, Moon JR, Berkman LF and Glymour MM (2012) Current and long-term spousal caregiving and onset of cardiovascular disease. Journal of Epidemiology and Community Health 66, 951-956.

Do YK and Malhotra C (2012) The effect of coresidence with an adult child on depressive symptoms among older widowed women in South Korea: an instrumental variable estimation. Journals of Gerontology: Psychological Sciences and Social Sciences 67B, 384-391.

Einiö E and Martikainen P (2019) Risk of hospitalization for cancer, musculoskeletal disorders, injuries, or poisonings surrounding widowhood. American Journal of Epidemiology 188, 110-118.

Elwert F and Christakis NA (2006) Widowhood and race. American Sociological Review 71, 16-41.

Fang SY, Huang N, Chen KH, Yeh HH, Lin KM and Chen CY (2012) Gender difference in widowhood effects among community-dwelling elders by causes of death in Taiwan. Annals of Epidemiology 22, 457-465. 
Gerra G, Monti D, Panerai AE, Sacerdote P, Anderlini R, Avanzini P, Zaimovic A, Brambilla F and Franceschi C (2003) Long-term immune-endocrine effects of bereavement: relationships with anxiety levels and mood. Psychiatry Research 121, 145-158.

Gove WR (1984) Gender differences in mental and physical illness: the effects of fixed roles and nurturant roles. Social Science \& Medicine 19, 77-91.

Gu D (2003) Analysis of gender differences in the effect of marriage on the health and longevity of the elderly in China. Chinese Journal of Population Science 3, 32-40. (In Chinese)

Helsing K and Szklo M (1981) Mortality after bereavement. American Journal of Epidemiology 114, 41-52.

Herm A, Anson J and Poulain M (2016) Living arrangements and marital status: a register-based study of survival of older adults in Belgium at the beginning of the 21st century. Ageing \& Society 36, 2141-2162.

Hu YR and Goldman N (1990) Mortality differentials by marital status: an international comparison. Demography 27, 233-250.

Huang Q, Du P and Chen G (2018) The intergenerational support between adult children and older adults and its associated factors. Population \& Development 24, 20-28.

Jones DA, Goldblatt PO and Leon D (1984) Bereavement and cancer: some data on deaths of spouses from the longitudinal study of Office of Population Censuses and Surveys. British Medical Journal (Clinical Research Edition) 289, 461-464.

Keene JR and Prokos AH (2008) Widowhood and the end of spousal care-giving: relief or wear and tear? Ageing \& Society 28, 551-570.

Kessler RC and Cleary PD (1980) Social class and psychological distress. American Sociological Review 45, 463-478.

Lee GR, DeMaris A, Bavin S and Sullivan R (2001) Gender differences in the depressive effect of widowhood in later life. Journals of Gerontology: Psychological Sciences and Social Sciences 56B, S56-S61.

Li L, Liang J, Toler A and Gu S (2005) Widowhood and depressive symptoms among older Chinese: do gender and source of support make a difference? Social Science \& Medicine 60, 637-647.

Li L, Zhang J and Liang J (2009) Health among the oldest-old in China: which living arrangements make a difference? Social Science \& Medicine 68, 220-227.

Liu H, Xiao Q, Cai Y and Li S (2015) The quality of life and mortality risk of elderly people in rural China: the role of family support. Asia Pacific Journal of Public Health 27, NP2232-NP2245.

Lund R, Due P, Modvig J, Holstein BE, Damsgaard MT and Andersen PK (2002) Cohabitation and marital status as predictors of mortality: an eight year follow-up study. Social Science \& Medicine 55, 673-679.

Manor O and Eisenbach Z (2003) Mortality after spousal loss: are there socio-demographic differences? Social Science \& Medicine 56, 405-413.

Martikainen P and Valkonen T (1996a) Mortality after death of a spouse in relation to duration of bereavement in Finland. Journal of Epidemiology and Community Health 50, 264-268.

Martikainen P and Valkonen T (1996b) Mortality after death of a spouse: rates and causes of death in a large Finnish cohort. American Journal of Public Health 86, 1087-1093.

McNally JW (2003) Health, widowhood, and family support in the north and south Pacific: a comparative study. Journal of Women \& Aging 15, 29-47.

Mellström D, Nilsson A, Oden A, Rundgren A and Svanborg A (1982) Mortality among widowed in Sweden. Scandinavian Journal of Social Medicine 10, 33-41.

Moon JR, Glymour MM, Vable AM, Liu S and Subramanian SV (2014) Short- and long-term associations between widowhood and mortality in the United States: longitudinal analyses. Journal of Public Health 36, 382-389.

Nielsen MK, Neergaard MA, Jensen AB, Bro F and Guldin MB (2016) Psychological distress, health, and socio-economic factors in caregivers of terminally ill patients: a nationwide population-based cohort study. Supportive Care in Cancer: Official Journal of the Multinational Association of Supportive Care in Cancer 24, 3057-3067.

Pezzin LE, Pollak RA and Schone BS (2007) Efficiency in family bargaining: living arrangements and caregiving decisions of adult children and disabled elderly parents. CESifo Economic Studies 53, 69-96.

Pimouguet C, Rizzuto D, Schön P, Shakersain B, Angleman A, Lagergren M, Fratiglioni L and Xu W (2016) Impact of living alone on institutionalization and mortality: a population-based longitudinal study. European Journal of Public Health 26, 182-187. 
Pizzetti P and Manfredini M (2008) The shock of widowhood? Evidence from an Italian population (Parma, 1989-2000). Social Indicators Research 85, 499-513.

Prior A, Fenger-Grøn M, Davydow DS and Olsen J (2018) Bereavement, multimorbidity and mortality: a population-based study using bereavement as an indicator of mental stress. Psychological Medicine 48, 1437-1443.

Santacroce A, Wastesson JW, Höhn A, Christensen K and Oksuzyan A (2018) Gender differences in the use of anti-infective medications before and after widowhood: a register-based study. Journal of Epidemiology \& Community Health 72, 526-531.

Schaefer C, Quesenberry CP and Wi S (1995) Mortality following conjugal bereavement and the effects of a shared environment. American Journal of Epidemiology 141, 1142-1152.

Schulz R, Newsom JT, Fleissner K, Decamp AR and Nieboer AP (1997) The effects of bereavement after family caregiving. Aging \& Mental Health 1, 269-282.

Shah SM, Carey IM and Harris T (2013) The effect of unexpected bereavement on mortality in older couples. American Journal of Public Health 103, 1140-1145.

Shah SM, Carey IM, Harris T, DeWilde S, Victor CR and Cook DG (2012) Do good health and material circumstances protect older people from the increased risk of death after bereavement? American Journal of Epidemiology 176, 689-698.

Shor E, Roelfs DJ, Curreli M, Clemow L, Burg MM and Schwartz JE (2012) Widowhood and mortality: a meta-analysis and meta-regression. Demography 49, 575-606.

Silverstein M and Bengtson VL (1991) Do close parent-child relations reduce the mortality risk of older parents? Journal of Health \& Social Behavior 32, 382-395.

Silverstein M and Bengtson VL (1994) Does intergenerational social support influence the psychological wellbeing of older parents? The contingencies of declining health and widowhood. Social Science \& Medicine 38, 943-957.

Skulason B, Jonsdottir LS, Sigurdardottir V and Helgason AR (2012) Assessing survival in widowers, and controls - a nationwide, six- to nine-year follow-up. BMC Public Health 12, 96.

Stahl ST, Arnold AM, Chen JY, Anderson S and Schulz R (2016) Mortality after bereavement: the role of cardiovascular disease and depression. Psychosomatic Medicine 78, 697-703.

Tiedt AD, Saito Y and Crimmins EM (2016) Depressive symptoms, transitions to widowhood, and informal support from adult children among older women and men in Japan. Research on Aging 38, 619-642.

Umberson D (1992) Gender, marital status and the social control of health behavior. Social Science o Medicine 34, 907-917.

Umberson D, Wortman CB and Kessler RC (1992) Widowhood and depression: explaining long-term gender differences in vulnerability. Journal of Health \& Social Behavior 33, 10-24.

Vable AM, Subramanian SV, Rist PM and Glymour MM (2015) Does the 'widowhood effect' precede spousal bereavement? Results from a nationally representative sample of older adults. American Journal of Geriatric Psychiatry 23, 283-292.

Walter-Ginzburg A, Blumstein T, Chetrit A and Modan B (2002) Social factors and mortality in the old-old in Israel: the CALAS study. Journals of Gerontology Psychological Sciences and Social Sciences 57B, S308-S318.

Wang G and Ge Y (2013) Status of widowed elderly population in China and its development trend. Scientific Research on Aging 1, 44-55. (In Chinese)

Ward RA (2008) Multiple parent-adult child relations and well-being in middle and later life. Journals of Gerontology: Psychological Sciences and Social Sciences 63B, S239-S247.

Williams BR, Zhang Y, Sawyer P, Mujib M, Jones LG, Feller MA, Ekundayo OJ, Aban IB, Love TE, Lott A and Ahmed A (2011) Intrinsic association of widowhood with mortality in community-dwelling older women and men: findings from a prospective propensity-matched population study. Journals of Gerontology: Biological Sciences and Medical Sciences 66A, 1360-1368.

Wright DM, Rosato M and O'Reilly D (2015) Urban/rural variation in the influence of widowhood on mortality risk: a cohort study of almost 300,000 couples. Health \& Place 34, 67-73.

Ytterstad E and Brenn T (2015) Mortality after the death of a spouse in Norway. Epidemiology 26, 289-294.

Cite this article: Liu H, Jiang Q, Feldman MW (2020). Widowhood and mortality risk of older people in rural China: do gender and living arrangement make a difference? Ageing \& Society 40, 1939-1955. https:// doi.org/10.1017/S0144686X19000436 